\title{
Magnetophotoluminescence of stacked self-assembled InP quantum dots
}

\author{
R. Provoost, ${ }^{\text {a) }}$ M. Hayne, and V. V. Moshchalkov \\ Laboratorium voor Vaste-Stoffysica en Magnetisme, Katholieke Universiteit Leuven, Celestijnenlaan 200 D, \\ B-3001 Leuven, Belgium \\ M. K. Zundel and K. Eberl \\ Max-Planck-Institut für Festkörperforschung, Heisenbergstrasse 1, 70569 Stuttgart, Germany
}

(Received 30 November 1998; accepted for publication 14 June 1999)

\begin{abstract}
We report magnetophotoluminescence measurements of stacked layers of self-assembled InP quantum dots. With a magnetic field applied in the growth direction we have determined the exciton reduced mass from the field dependence of the photoluminescence energy. By applying a magnetic field perpendicular to the growth direction, we have analyzed the spatial confinement of the dots in the growth direction. We observe a large increase in the shift of the exciton energy between 0 and $50 \mathrm{~T}$ when the thickness of the GaInP spacer layer between the dots is reduced from 8 to $4 \mathrm{~nm}$. This indicates a decrease in spatial confinement in the growth direction which we attribute to strong electronic coupling between vertically stacked dots. (C) 1999 American Institute of Physics.
\end{abstract} [S0003-6951(99)03832-2]

During the last few years much effort has been put into the study of self-assembled semiconductor quantum dots (QDs), due to their promising optical and electrical properties for applications such as lasers. ${ }^{1}$ Photoluminescence (PL) measurements on these QDs reveal an intense emission of visible $(\operatorname{InP})^{2-4}$ or infrared $\left[\operatorname{In}(\mathrm{Ga}) \mathrm{As},{ }^{1,5-9} \operatorname{InSb},{ }^{10}\right.$ and InAlAs $]^{11}$ light, with linewidths of the order of $40 \mathrm{meV}$, from the confined excitons in the dots. These rather broad lines are caused by the luminescence of large amounts of QDs, $\sim 10^{10} \mathrm{~cm}^{-2}$, with a typical $10 \%$ size nonuniformity. On the other hand, by repeating layers of dots separated by a few nanometers a vertical alignment occurs giving a reduction in the linewidth due to a more homogeneous size distribution and electronic coupling. ${ }^{3,5,6}$ Here we present PL measurements of stacked InP QDs in magnetic fields up to $50 \mathrm{~T}$. Our data show that reducing the thickness of the GaInP barrier between layers of dots results in strong electronic coupling between vertically stacked InP dots. The control of the electronic coupling of QDs offers the potential for wavefunction engineering, which is interesting, not only for fundamental physics, ${ }^{12}$ but also for laser applications. Indeed, the first injection laser based on stacked InP quantum dots has recently been reported. ${ }^{13}$

The InP quantum dots were grown by solid-source molecular beam epitaxy on a GaAs substrate with a GaAs buffer layer, and embedded in a $\mathrm{Ga}_{0.52} \mathrm{In}_{0.48} \mathrm{P}$ waveguide layer. The lattice mismatch between the InP and the $\mathrm{Ga}_{0.52} \mathrm{In}_{0.48} \mathrm{P}$ is $3.7 \%$. For sample A, only one layer of dots was grown by depositing nominally $3 \mathrm{ML}$ of InP. For samples B, C, and D three layers of InP dots were grown, separated by GaInP spacer layers with nominal thicknesses of 8,4 , and $2 \mathrm{~nm}$, respectively. A detailed description of these samples can be found elsewhere. ${ }^{3}$ Cross-sectional transmission electron microscopy (TEM) pictures show that for samples B, C and D the InP QDs are nicely stacked above each other. ${ }^{3}$ The dots

${ }^{\text {a)} E l e c t r o n i c ~ m a i l: ~ r i k . p r o v o o s t ~ @ ~ f y s . k u l e u v e n . a c . b e ~}$ have typical dimensions of $16 \mathrm{~nm}$ diameter and about $2 \mathrm{~nm}$ height, with a density of $5 \times 10^{10} \mathrm{~cm}^{-2}$ per layer. The PL experiments were performed at $4.2 \mathrm{~K}$ in pulsed magnetic fields up to $50 \mathrm{~T}$, applied parallel and perpendicular to the growth direction, $z$. The excitation was provided by a continuous wave frequency-doubled solid-state laser operating at $532 \mathrm{~nm}$, and transmitted to the sample through an optical fiber. The diameter of the illuminated region was about 2 $\mathrm{mm}$ with a maximum laser power of $20 \mathrm{~mW}$. The PL was collected by six pick-up fibers surrounding the excitation fiber, dispersed in a monochromator with a 150 grooves $/ \mathrm{mm}$ grating, and projected on an intensified charge coupled device detector. By measuring the $\mathrm{PL}$ at the maximum of the $20 \mathrm{~ms}$ magnetic field pulse, a constant field within $\pm 1 \%$ was obtained during an exposure time of $1.8 \mathrm{~ms}$. Additional data were obtained during the down sweep of the pulse using an exposure time of $0.3 \mathrm{~ms}$ ( $\pm 3 \%$ field variation).

In Fig. 1 we present the evolution of the PL energy as a function of magnetic field $(B)$. The energy of the PL line is given by the center of mass of the peak as determined from numerical analysis. ${ }^{14}$ Despite the large linewidths (up to 43 $\mathrm{meV}$ ), the center of mass allows us to determine energy shifts of the PL to within $1 \mathrm{meV}$ accuracy. A separate analysis of the shape of the PL line shows it to be a single Gaussian with a linewidth that does not change significantly with magnetic field $(<1 \mathrm{meV})$. The zero-field PL energy redshift with decreasing thickness of the GaInP spacer layers has been ascribed to the reduction of compressive strain in the densely stacked dots and, eventually, to strong electronic coupling. ${ }^{3}$ This is consistent with our results. The small offset observed for the zero-field PL peak positions for the two field directions in Fig. 1, is due to the difference in the position of the laser spot on the sample for the two field configurations. Magnetophotoluminescence measurements on different positions on the sample reveal almost identical energy shifts with field. The shifts of the PL energy between 0 and $50 \mathrm{~T}$ are given in Table I for both field configurations. 


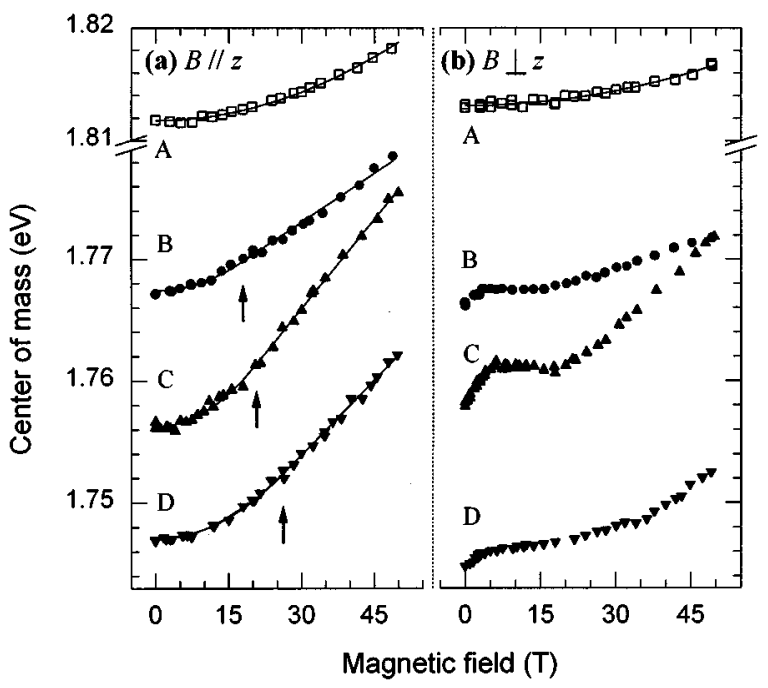

FIG. 1. Center of mass of the PL energy at $4.2 \mathrm{~K}$ with magnetic fields parallel (a) and perpendicular (b) to the growth direction for the sample with a single layer of InP dots (A), and for the samples with three layers of dots separated by 8,4 , and $2 \mathrm{~nm}(\mathrm{~B}, \mathrm{C}$, and $\mathrm{D}$, respectively). The arrows indicate the crossover from parabolic to linear field dependence.

In the low-magnetic-field regime, the electron and hole wavefunctions are perturbed resulting in a diamagnetic shift of the energy levels. Conventionally, the PL energy shift is quadratic with magnetic field and given by $\Delta E$ $=e^{2}\left\langle\rho^{2}\right\rangle B^{2} / 8 \mu$. Here $\mu$ is the exciton reduced mass and $\sqrt{\left\langle\rho^{2}\right\rangle}$ is the exciton radius in the plane perpendicular to the magnetic field direction. This parabolic field dependence has been seen in many studies on single layers of self-assembled QDs. ${ }^{8-11}$ In sufficiently large magnetic fields, the magnetic length can become smaller than the dot diameter, and the magnetic rather than the spatial confinement of the excitons dominates. In this regime the energy shift is linear in field, $\Delta E=\frac{1}{2} \hbar \omega_{c}=(\hbar e / 2 \mu) B$, parallel with the lowest Landau level.

The lines through the data points in Fig.1(a) are leastsquare fits to the data with a parabola at low fields and a straight line at high fields for samples B, C, and D. By including the restriction that the gradient of the fit matches at the crossover point, this analysis has three fitting parameters which yield zero-field position, exciton radius $\left(\sqrt{\left\langle\rho^{2}\right\rangle}\right)$ and exciton mass $(\mu)$. We could not distinguish a linear part in the data for sample A; therefore the line through these data is a parabola. From the slope of the linear parts of samples B,

TABLE I. Details of the InP QD samples A, B, C, and D: the stack height for the samples with three layers of dots, the PL linewidth, the shift of the PL line between 0 and $50 \mathrm{~T}$ for fields parallel to $z$, the exciton mass, the exciton radius in the plane of the layers, and the shift of the PL line between 0 and $50 \mathrm{~T}$ for fields perpendicular to $z$. The errors determined from the fit are given between brackets for $\mu$ and $\sqrt{\left\langle\rho^{2}\right\rangle}$.

\begin{tabular}{ccccccc}
\hline \hline Sample & $\begin{array}{c}\text { Stack height } \\
(\mathrm{nm})\end{array}$ & $\begin{array}{c}\text { Linewidth } \\
(\mathrm{meV})\end{array}$ & $\begin{array}{c}B \|_{z} \\
(\mathrm{meV})\end{array}$ & $\begin{array}{c}\mu \\
\left(\mathrm{m}_{e}\right)\end{array}$ & $\begin{array}{r}\sqrt{\left\langle\rho^{2}\right\rangle} \\
(\mathrm{nm})\end{array}$ & $\begin{array}{c}B \perp z \\
(\mathrm{meV})\end{array}$ \\
\hline A (single) & $\ldots$ & 43 & 6.3 & & & 3.6 \\
$\mathrm{~B}(8 \mathrm{~nm})$ & 20 & 40 & 11.4 & $0.22(2)$ & $8.6(4)$ & 5.5 \\
$\mathrm{C}(4 \mathrm{~nm})$ & 12 & 28 & 19.4 & $0.12(1)$ & $8.0(2)$ & 13.8 \\
$\mathrm{D}(2 \mathrm{~nm})$ & 8 & 24 & 15.2 & $0.14(1)$ & $7.1(1)$ & 7.7 \\
\hline \hline
\end{tabular}

$\mathrm{C}$, and $\mathrm{D}$ we have determined the reduced mass of the excitons: $0.22,0.12$, and $0.14 \mathrm{~m}_{e}$ respectively (Table I). The exciton masses are large compared with the InP bulk value $\left(0.0678 \mathrm{~m}_{e}\right)$, which can be attributed to the effects of compressive strain and overlap of the hole wavefunction into the GaInP. ${ }^{15}$ Enhancements of the mass have also been observed in magneto-optical studies of single InAs QDs. ${ }^{8}$ From the parabolic field dependence of the PL energy we have determined the exciton radii in the plane of the layers using the respective values for $\mu$ (Table I). Calculations of electron and hole wavefunctions in strained self-organized InP dots indicate that the small negative valence band offset results in confinement of the holes, partly outside the dots, entirely due to the effect of strain. ${ }^{15}$ The vertical stacking of QDs is a process in which the strain field of lower layers tends to provoke the formation of vertically aligned dots in the upper layers. The large redshift in the zero-field PL peak is strong evidence that this process also relaxes the strain within the dots themselves. The variations in the exciton mass and radius seen in our data suggest that as this happens changes in the hole confinement are induced varying the wavefunction penetration into the GaInP. Definite conclusions cannot be drawn about the exciton properties in a single layer of dots, since no linear behavior is observed. However, this very fact requires that the exciton radius is consistently smaller than for the stacked layer samples. This implies a significant difference in the way in which carriers are confined in single compared to stacked layers.

For magnetic fields in the plane of the layers, the confinement of the electrons and holes is probed in the growth direction, i.e., the smallest dimension of the dots. For sample A, we observe a conventional parabolic behavior of the PL energy [Fig. 1(b)]. The shift is only $3.6 \mathrm{meV}$ which illustrates the strong spatial confinement in the $z$ direction. A slightly larger shift is observed for sample B with three layers of stacked dots, but for sample $\mathrm{C}$ a remarkable deviation is observed. The shift increases by a factor of 2.5 compared to sample B in this field orientation. This reflects a decrease in the confinement of the system in the growth direction. TEM studies show no large differences in size and shape distribution of the dots for samples B and C. Moreover, the field dependence of the PL energy clearly deviates strongly from conventional parabolic and then linear behavior, indicative of anomalous electronic structure within the sample. A very steep increase of the PL energy is observed below $5 \mathrm{~T}$, hardly any shift is seen between 5 and $20 \mathrm{~T}$ and above $20 \mathrm{~T}$ the PL energy shifts again to higher energies. Notably, the size of the shift decreases again for sample D, but the peculiar movement of the PL line remains. Although the shift is very small for sample $\mathrm{B}$, unconventional behavior at low fields can also be observed.

We believe that the results obtained with the field perpendicular to $z$ are a result of strong electronic coupling between vertically stacked QDs in samples C and D. The fact that the shift of the line for sample D is significantly smaller than for sample $\mathrm{C}$ can be explained by examining the total height of the stack of dots for the two samples. For strongly coupled dots the vertical confinement is no longer given by the height of a single dot, but by the total stack height of the InP dots. Sample $C$ has a total stack height of $12 \mathrm{~nm}$ and a 


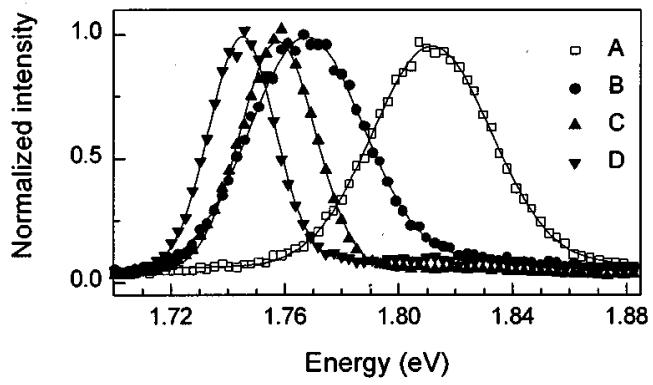

FIG. 2. PL spectra recorded in $1.8 \mathrm{~ms}$ at zero field and $4.2 \mathrm{~K}$ for the sample with a single layer of InP dots $(\square)$, and for the three samples with three layers of dots spaced by $8(\mathbf{\bullet}), 4(\mathbf{\Lambda})$, and $2 \mathrm{~nm}(\boldsymbol{\nabla})$ of GaInP. Note a substantial decrease of the linewidth as the layer separation is reduced. (The lines are Gaussian fits.)

shift of $13.8 \mathrm{meV}$, whereas the reduced layer separation for sample D gives a stack height of $8 \mathrm{~nm}$, increased confinement, and a correspondingly smaller shift of $7.7 \mathrm{meV}$. Sample B has a much larger stack height $(20 \mathrm{~nm})$, but a smaller shift, implying that the dots are not strongly coupled. The unconventional low field behavior of sample B which is very similar to samples $\mathrm{C}$ and $\mathrm{D}$, could therefore have its origin in a weak coupling between the layers, but one which is destroyed at high magnetic fields, such that the overall shift is small.

The strong coupling of the dots also produces a dramatic decrease in the linewidth as indicated in Table I and Fig. 2. This is because relative size fluctuations in the total height of the stacked dots are much smaller than for a single layer, resulting in a more uniform electronic system. ${ }^{3,5,6}$ Furthermore, it is consistent with the dots in samples $\mathrm{C}$ and $\mathrm{D}$ being strongly coupled, but not sample B.

In conclusion, we have studied the magnetic field dependence of the PL of InP QDs in a single layer and in stacked layers of vertically aligned dots. We have observed that reducing the barrier thickness between the dots leads to a decrease of the confinement of the excitons. This is a clear evidence for the onset of strong electronic coupling between the vertically aligned dots. We have also obtained a much larger exciton mass than for bulk InP, indicating the large strain effect and an overlap of the wavefuntions in the GaInP barrier. A better understanding of the effects of electronic coupling between vertically aligned dots is very important and can be very promising for improving the properties of self-assembled QDs for laser applications.

This research has been supported by the FWOVlaanderen, the GOA and the IUAP programs. M.H. is a fellow of the Research Council of the KU Leuven. The authors are grateful to F. M. Peeters and L. Eaves for helpful discussions.

${ }^{1}$ D. Bimberg, N. N. Ledentsov, M. Grundmann, F. Hinrichsdorff, V. M. Ustinov, P. Kop'ev, Zh. I. Alferov, and J. A. Lott, Solid-State Electron. 42, 1433 (1998).

${ }^{2}$ A. Kurtenbach, C. Ulrich, N. Y. Jin-Phillipp, F. Noll, K. Eberl, K. Syassen, and F. Phillipp, J. Electron. Mater. 25, 395 (1996).

${ }^{3}$ M. K. Zundel, P. Specht, K. Eberl, N. Y. Jin-Phillipp, and F. Phillipp, Appl. Phys. Lett. 71, 2972 (1997).

${ }^{4}$ B. Kowalski, S. Nomura, C. Pryor, Y. Aoyagi, N. Carlsson, M.-E. Pistol, P. Omling, L. Samuelson, and W. Seifert, Phys. Rev. B 58, 2026 (1998).

${ }^{5}$ G. S. Solomon, J. A. Trezza, A. F. Marshall, and J. S. Harris, Jr., Phys. Rev. Lett. 76, 952 (1996).

${ }^{6}$ R. Heitz, A. Kalburge, Q. Xie, M. Grundmann, A. Hoffmann, A. Madhukar, and D. Bimberg, Phys. Rev. B 57, 9050 (1998).

${ }^{7}$ I. E. Itskevich, M. Henini, H. A. Carmona, L. Eaves, P. C. Main, D. K. Maude, and J. C. Portal, Appl. Phys. Lett. 70, 505 (1997).

${ }^{8}$ R. K. Hayden, K. Uchida, N. Miura, A. Polimeni, S. T. Stoddart, M. Henini, L. Eaves, and P. C. Main, Physica B 249-251, 262 (1998).

${ }^{9}$ R. Rinaldi, R. Mangino, R. Cingolani, H. Lipsanen, M. Sopanen, J. Tulkki, M. Braskeń, and J. Ahopelto, Phys. Rev. B 57, 9743 (1998).

${ }^{10}$ E. Alphandéry, R. J. Nicholas, N. J. Mason, B. Zhang, P. Möck, and G. R. Booker, Appl. Phys. Lett. 74, 2041 (1999).

${ }^{11}$ P. D. Wang, J. L. Merz, S. Fafard, R. Leon, D. Leonard, G. MedeirosRibeiro, M. Oestreich, P. M. Petroff, K. Uchida, N. Miura, H. Akiyama, and H. Sakaki, Phys. Rev. B 53, 16458 (1996).

${ }^{12}$ G. Schedelbeck, W. Wegscheider, M. Bichler, and G. Abstreiter, Science 278, 1792 (1998).

${ }^{13}$ M. K. Zundel, N. Y. Jin-Phillipp, F. Phillipp, K. Eberl, T. Riedl, E. Fehrenbacher, and A. Hangleiter, Appl. Phys. Lett. 73, 1784 (1998).

${ }^{14}$ I. V. Kukushin, N. J. Pulsford, K. von Klitzing, K. Ploog, and V. B. Timofeev, Surf. Sci. 263, 30 (1992).

${ }^{15}$ C. Pryor, M-E. Pistol, and L. Samuelson, Phys. Rev. B 56, 10404 (1997). 